Research Article

\title{
Health service utilization in Manicaland Province, Zimbabwe during the COVID-19 pandemic: results from a cross-sectional household survey
}

\author{
Julia Scott', Melissa S Nolan', Joseph Mberikunashe², Oscar Tapera ${ }^{3}$, Mufaro Kanyangarara' ๑ \\ 1 Department of Epidemiology and Biostatistics, Arnold School of Public Health, University of South Carolina, Columbia, South Carolina, USA, \\ 2 National Malaria Control Program, Ministry of Health and Child Care, Harare, Zimbabwe, ${ }^{3}$ Sadtap Health Research Institute, Harare, Zimbabwe \\ Keywords: COVID-19, health care utilization, essential health services, Zimbabwe, sub-Saharan Africa \\ https://doi.org/10.29392/001c.31599
}

\section{Journal of Global Health Reports}

Vol. 6, 2022

\begin{abstract}
Background
The coronavirus disease 2019 (COVID-19) pandemic has impacted utilization of health care, especially in low-resource settings in Sub-Saharan Africa. Here, the current state of health care utilization in Manicaland Province, Zimbabwe during the COVID-19 pandemic was assessed.
\end{abstract}

\begin{abstract}
Methods
Cross-sectional surveys were administered to randomly selected households in three districts in Manicaland Province, Zimbabwe from August to September 2020. Data on socio-demographic characteristics, utilization and perceptions of health facilities, and COVID-19 risk perceptions and impact were collected using a structured questionnaire administered to heads of households. Factors associated with health care utilization in the 4 weeks prior to the survey were identified using logistic regression.
\end{abstract}

\section{Findings}

Among the 542 respondents enrolled in the study, most were married or co-habiting, were employed, had attended secondary school or higher, and headed households with more than 4 members. About half (53.3\%) of households reported visiting a health facility in the previous 4 weeks. The most cited barriers of service at health facilities were the unavailability of medicines, cost of services and waiting time see a provider. The multivariate logistic regression analysis indicated that the likelihood of health care utilization in the previous 4 weeks was higher among households with a respondent who was 55 years or older, had attended secondary school or higher, and was unemployed; and among households with a child under 5 years or a pregnant woman and located in Makoni or Mutare rural districts.

\section{Conclusions}

These findings indicate the need to improve quality of care by increasing the availability of essential medicines, removing financial barriers to health services, and reducing waiting times. Key factors such as age and education level of the head of household and the presence of vulnerable populations such as children and pregnant women in households should be considered when targeting health awareness and education to improve health service utilization during the pandemic.

The recent coronavirus disease 2019 (COVID-19) pandemic has impacted health systems globally since it was declared a public health emergency of international concern by the World Health Organization (WHO) in January $2020 .^{1}$ Various COVID-19 mitigation strategies such as lockdowns, curfews and stay-at-home policies have been implemented to curb transmission. However, these measures have resulted in widespread disruptions in essential health ser- vices. Over $90 \%$ of countries have reported disruptions in essential health services. ${ }^{2}$ Several mathematical models have attempted to estimate the indirect impact of COVID-19-related disruptions on essential health service provision and have projected increases in overall and cause-specific mortality. ${ }^{3-5}$ For instance, one model estimated that drops in coverage of essential reproductive, maternal, newborn, child and adolescent health and nutrition 
interventions of up to $20 \%$ over 6 months would result in an excess of 253,500 child deaths and 12,200 maternal deaths globally. ${ }^{5}$ More severe drops in coverage of up to $50 \%$ were predicted to result in over 1 million additional child deaths and 56,700 additional maternal deaths globally. ${ }^{5}$ Sub-Saharan Africa is particularly vulnerable to the indirect mortality effects of the pandemic due to limited resources, fragile health systems, weak surveillance systems, political instability, and endemic diseases such as malaria. ${ }^{6,7}$ Recent reports indicate worrisome drops in outpatient visits in 2020 compared to $2019 .^{8}$

The decision to seek care is often influenced by perceived susceptibility to disease or illness, perceived severity of a disease or illness, perceived benefits of utilizing health care, perceived barriers to utilizing health care, cues to action, and self-efficacy. ${ }^{9}$ Several studies in sub-Saharan Africa have identified factors associated with health care utilization, including age, education, rural residence, distance to the nearest health facility, knowledge, risk perceptions and perceived and actual quality of care. ${ }^{10-13}$ Even when care is sought, appropriate care may not be received due to lack of trained personnel and relevant guidelines, unavailability of equipment, essential medicines and commodities, and limited diagnostic capacity. ${ }^{14}$ This can result in a growing number of untreated illnesses, and both COVID-19 and non-COVID-19 related deaths.

During the 2014-2016 Ebola Virus Disease (EVD) outbreak in West Africa, excess non-EVD morbidity and mortality were observed. ${ }^{15-19}$ Access to essential health services was limited due to the closure of health facilities and inability of operational health facilities to see patients. ${ }^{15,16}$ Fear of contracting EVD led to an 18\% decrease in the use of essential health services in areas with lower EVD incidence and a $27 \%$ decrease in areas with higher EVD incidence. ${ }^{17}$ The number of all-cause deaths increased during the EVD outbreak compared to previous years. ${ }^{18}$ Notably, gaps in the provision of services for malaria, tuberculosis, and human immunodeficiency virus (HIV) during the outbreak led to excess mortality from these endemic diseases. ${ }^{19}$ Some studies postulate that declines in health care utilization and limited service provision resulted in additional nonEVD mortality in excess of the actual EVD mortality observed during the outbreak. ${ }^{19-21}$

It is essential to understand the current state of health care utilization in the context of the COVID-19 pandemic to reduce the potential indirect mortality effects of the pandemic. The overall goal of this study was to identify barriers influencing the use of essential health services and assess factors associated with household health care utilization in the previous 4 weeks in Manicaland Province, Zimbabwe during the COVID-19 pandemic. Immediately following the detection of the first case of COVID-19 in Zimbabwe in March 2020, the government implemented a nationwide lockdown, in which stay-at-home orders and curfews were implemented. ${ }^{22}$ Even prior to the pandemic, the health care system in Zimbabwe was not faring well with limited hospital beds and equipment, shortages in health care workers, frequent industrial actions by health care workers and stock-outs of essential medicines and commodities. ${ }^{23}$
COVID-19-related disruptions in supply chains, diversion of workers to the COVID-19 response, and the suspension of elective health services have hindered the provision of essential health services delivery, further overwhelming the fragile heath system. ${ }^{22-25}$ The stigma and fear surrounding COVID-19 have also caused many to delay or completely avoid seeking care. ${ }^{25}$ Understanding health care utilization patterns amid the pandemic remains crucial to both addressing the pandemic and ensuring the pandemic does not inadvertently impact other health outcomes.

\section{METHODS}

\section{STUDY SETTING AND DESIGN}

A cross-sectional, community-based household survey was conducted in Manicaland Province, Zimbabwe from August 31, 2020 to September 10, 2020, during the first peak of confirmed COVID-19 cases in Zimbabwe (Figure 1). Manicaland Province, which lies on the border with Mozambique, has a population of about $1,780,000$ residents. ${ }^{26}$ The province is divided into 7 administrative districts. There are 277 health facilities serving the predominantly rural population, and most health facilities (89\%) are governmentowned rural health centers and clinics. ${ }^{27}$ The outpatient department visit rate for the province averages 19 visits per 100 people. On August 31, 2020, when the survey started, Manicaland Province had 376 cumulative COVID-19 cases, 19 COVID-19 deaths and the highest provincial case fatality rate $^{28}$ The start of the survey also coincided with the relaxation of nationwide lockdown that commenced in March 2020, curfews were narrowed, and business hours extended. $^{22}$ Restrictions on social gatherings and enforcement of mask-wearing remained in place.

A total of 547 households in Makoni, Mutare rural, and Buhera districts were randomly selected using a sampling frame, based on the 2012 Zimbabwe Population Census. ${ }^{26}$ The sample size required was based on detecting a $15 \%$ difference in health care utilization with a $95 \%$ confidence level, $5 \%$ level of precision and non-response rate of $5 \%$. Makoni, Mutare rural and Buhera districts were purposively chosen to represent areas of high, moderate, and low malaria transmission, respectively. Heads of selected households were eligible to participate if they were 18 years or older, and willing and able to provide consent. Households were visited up to three times if the household head (respondent) was not available. A standardized questionnaire available in English and Shona (the local language) was administered face-to-face using smartphones to collect information on demographics, socioeconomic status, knowledge of malaria and COVID-19, use of prevention measures for malaria and COVID-19, and health care utilization. To understand the barriers that might influence utilization of health services, the survey contained questions on perceptions of the quality of care at heath care facilities. The barriers to service assessed were time waiting to see a provider, ability to discuss problems or concerns about health, amount of explanation received about problem or treatment, privacy from having others see the examination, privacy from having others hear consultation, 


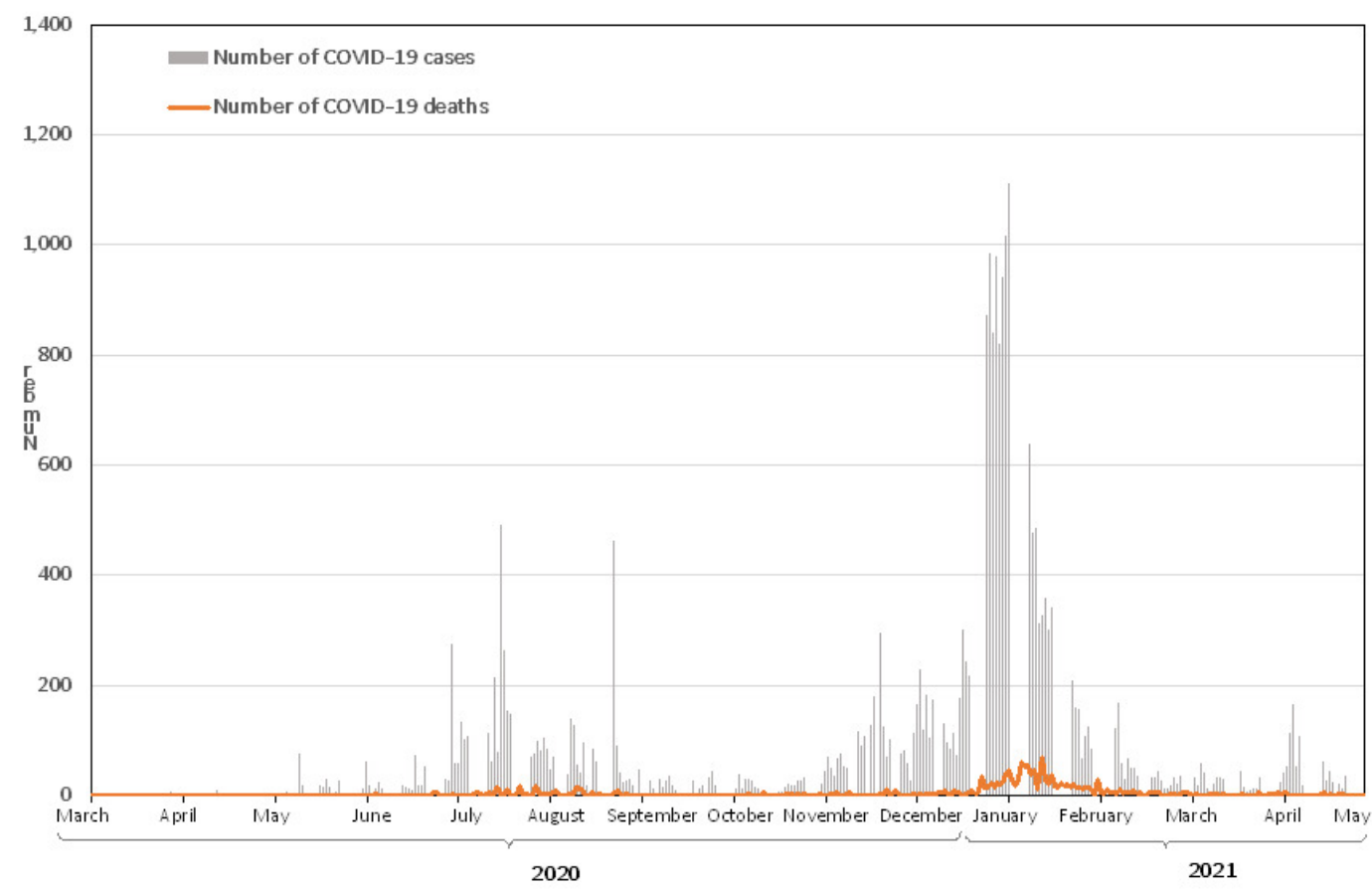

Figure 1. Confirmed COVID-19 cases and deaths in Zimbabwe, March 2020 - May 2021.

availability of medication, hours of service, number of days services are available, cleanliness of the facility, treatment by staff, and cost of services. Respondents were asked to indicate on a 3 point Likert scale whether each barrier was a "major problem", "minor problem" or "no problem".

\section{STATISTICAL ANALYSIS}

The outcome of interest, health care utilization, was based on responses to the question: "When was the last time you or another member of your household visited the health facility for care?" Responses were categorized into two groups: < 4 weeks ago, and $\geqslant 4$ weeks ago. Factors assessed for an association with the outcome included socio-demographic characteristics of the respondent: age (18-29, $30-39,40-54$, and $\geqslant 55$ years), marital status (married/cohabiting and not married which included divorced, separated, widowed, and never married), highest level of education attended (primary school or lower, secondary school or higher), and employment status (employed, unemployed). Household characteristics considered were household size ( $\leqslant 4$ household members, $>4$ household members), having at least one child less than 5 years living in the household (yes, no), having at least one pregnant woman living in the household (yes, no), travel minutes to the health facility as a proxy for access to care and household wealth index as a proxy for socioeconomic status. A household wealth index was calculated using principal components analysis, based on ownership of a radio, television, refrigerator, cellphone, solar panels, computer, stereo, cows, mules, goats, pigs, bicycle, car, and motorcycle; source of drinking water; type of toilet and main source of energy. The resulting index was divided into tertiles to represent the poorest, middle, and wealthiest households. For questions related to service barriers, dichotomous variables were created to indicate problem (major and minor) compared to no problem. Information on concern about the spread of COVID-19 and infection; and loss of household income due to the COVID-19 pandemic were included. Respondents who answered "Don't know" for the outcome or independent variables of interest were excluded from the present analysis $(\mathrm{n}=5)$. The final analytical sample included responses from 542 household heads.

Descriptive statistics were performed to characterize sampled households. Unadjusted and adjusted odds ratios (OR and aOR), corresponding 95\% confidence intervals (CI), and $\mathrm{p}$-values were obtained from univariate and multivariate logistic regression. The final multivariate model was fitted to the outcome using backwards elimination variable selection with an alpha less than 0.1 . This model was compared to the final model using the forwards selection approach. The log likelihood ratio test was used to assess goodness of fit. Variance inflation factors (VIF) were used to assess multicollinearity. A P value less than 0.05 was considered statistically significant. Statistical analyses were conducted using SAS 9.4 software.

\section{ETHICS CONSIDERATIONS}

Ethical approval was obtained from the Medical Research Council of Zimbabwe (MRCZ/A/2633). Before enrollment, written informed consent was obtained from the respondents. 
Table 1. Characteristics of households in Manicaland Province, Zimbabwe (N=542).

\begin{tabular}{|c|c|}
\hline Characteristics & $\mathrm{n}(\%)$ \\
\hline \multicolumn{2}{|l|}{ Age of respondent } \\
\hline $18-29$ years & $136(25.1)$ \\
\hline 30-39 years & $136(25.1)$ \\
\hline $40-54$ years & $149(27.5)$ \\
\hline$\geq 55$ years & $121(22.3)$ \\
\hline \multicolumn{2}{|l|}{ Marital status of respondent } \\
\hline Not married & $148(27.3)$ \\
\hline Married/co-habiting & $394(73.6)$ \\
\hline \multicolumn{2}{|l|}{ Highest level of education attended by respondent } \\
\hline Primary school or lower & $194(35.8)$ \\
\hline Secondary school or higher & $348(64.2)$ \\
\hline \multicolumn{2}{|l|}{ Employment status of respondent } \\
\hline Employed & $356(65.7)$ \\
\hline Unemployed & $34.3(186)$ \\
\hline \multicolumn{2}{|l|}{ District } \\
\hline Buhera & 211 (38.9) \\
\hline Makoni & $115(21.2)$ \\
\hline Mutare rural & $216(39.9)$ \\
\hline \multicolumn{2}{|l|}{ Household size } \\
\hline$\leq 4$ household members & $227(41.5)$ \\
\hline$>4$ household members & $320(58.5)$ \\
\hline \multicolumn{2}{|l|}{ Household wealth } \\
\hline Poorest & $181(33.4)$ \\
\hline Middle & $181(33.4)$ \\
\hline Wealthiest & $180(33.2)$ \\
\hline Had health insurance & $22(4.2)$ \\
\hline At least one child less than 5 years living in the household & $343(63.3)$ \\
\hline At least one pregnant woman living in the household & $73(13.5)$ \\
\hline
\end{tabular}

\section{RESULTS}

\section{CHARACTERISTICS OF HOUSEHOLDS}

Among the 542 households included, most respondents were married or co-habiting (73.6\%), had completed secondary school or higher (64.2\%), were employed $(65.7 \%)$ and were less than 55 years of age (77.7\%) (Table 1). More than half $(58.5 \%)$ of households had more than 4 members, with $63.3 \%$ reporting at least one child less than 5 years of age and $13.5 \%$ at least one pregnant woman living in the household. Fewer (21.2\%) households were located in Makoni district compared to Buhera and Mutare rural districts (38.9\% and $39.9 \%$ respectively). The median time to travel to a health facility was 30 minutes.

\section{PERCEIVED BARRIERS TO HEALTH SERVICE AND SUSCEPTIBILITY TO COVID-19}

Respondents were asked about their perception of the quality of services at their health facility, specifically the poten- tial service barriers they perceived to be a problem (Table 2). Among the service barriers considered, the availability of medicines at the health facility was the most commonly cited as a problem (57.0\%), followed by the cost of services or treatment $(19.4 \%)$, the time waited to see a provider (17.9\%), and the availability of personnel (17.3\%). Very few respondents perceived the cleanliness of the health facility (3.5\%) and the number of days services were available (6.8\%) to be a problem. Some respondents were very concerned or concerned about COVID-19 spread in the community (28.2\%) and COVID-19 infection (38.9\%). By contrast, $49.2 \%$ and $39.1 \%$ of respondents were not concerned at all about the spread of COVID-19 in the community or becoming infected with COVID-19 respectively. Most households reported experiencing a loss of household income due to the COVID-19 pandemic (88.2\%), with $67.9 \%$ reporting a complete loss of household income. 
Table 2. Perceptions of health services, COVID-19 risk perceptions and impact in Manicaland Province, Zimbabwe (N=542).

\begin{tabular}{|c|c|}
\hline Perceived problems at health facility & $\mathrm{n}(\%)$ \\
\hline Availability of medicines at facility & $309(57.0)$ \\
\hline Cost of services or treatment & $105(19.4)$ \\
\hline Time waited to see a provider & $97(17.9)$ \\
\hline Availability of personnel at facility & $94(17.3)$ \\
\hline Treatment by staff & $92(17.0)$ \\
\hline Amount of explanation received about the problem or treatment & $71(13.1)$ \\
\hline Visual privacy of examination & $67(12.4)$ \\
\hline Auditory privacy of consultation discussion & $65(12.0)$ \\
\hline Hours of service at facility & $65(12.0)$ \\
\hline Ability to discuss problems or concerns about health & $63(11.6)$ \\
\hline Number of days services are available & $37(6.8)$ \\
\hline Cleanliness of the facility & $19(3.5)$ \\
\hline \multicolumn{2}{|l|}{ COVID-19 risk perceptions and impact } \\
\hline \multicolumn{2}{|l|}{ Concern about COVID-19 spread in community } \\
\hline Very concerned & $82(15.1)$ \\
\hline Concerned & $71(13.1)$ \\
\hline A little concerned & $124(22.9)$ \\
\hline Not concerned & $265(48.9)$ \\
\hline \multicolumn{2}{|l|}{ Concerned about COVID-19 infection } \\
\hline Very concerned & $130(24.0)$ \\
\hline Concerned & $81(14.9)$ \\
\hline A little concerned & $119(22.0)$ \\
\hline Not concerned & $212(39.1)$ \\
\hline \multicolumn{2}{|l|}{ Loss of household income experience due to COVID-19 pandemic } \\
\hline None & $64(11.8)$ \\
\hline Partial & $110(20.3)$ \\
\hline Complete & 368 (67.9) \\
\hline
\end{tabular}

\section{FACTORS ASSOCIATED WITH HEALTH CARE UTILIZATION}

About half (53.3\%) of households reported using health care services in the previous 4 weeks. In the univariate logistic regression analysis, factors significantly associated with health care utilization in the previous 4 weeks were highest level of education attended and employment status of respondents, district of residence, having at least one child under the age of 5 and one pregnant woman living in the household (Table 3). The univariate analysis also indicated that citing concern about the spread of COVID-19 in the community and problems with privacy from having others hear the consultation, availability of personnel at the facility, and treatment by staff increased the likelihood of health care utilization in the previous 4 weeks. VIF estimates did not detect multicollinearity between independent variables. Results using backwards elimination and forwards selection yielded identical results. Significant factors associated with health care utilization in the multivariate analysis in the previous 4 weeks were education completed by the respondent, district, and having at least one child under the age of 5 and at least one pregnant woman living in the household (Table 3). Respondents who had attended secondary school or higher, were unemployed or were 55 years or older had a significantly higher likelihood of utilizing health care in the previous 4 weeks compared to others (adjusted odds ratio aOR 2.16; 95\% confidence interval [1.36-3.43]; 1.63 [1.11-2.41]; 1.89 [0.99-3.61]). Those living in a household with a child under 5 years, or a pregnant woman had more than double the odds of utilizing health care in the previous 4 weeks (2.35 [1.59-3.46]; 2.22 [1.26-3.93] respectively). Those living in Makoni and $\mathrm{Mu}$ tare rural districts had increased odds of utilizing health care in the previous 4 weeks compared to those living in Buhera district (2.35 [1.44-3.84]; 2.57 [1.71-3.92]). After controlling for other variables, none of the variables related to perceived problems at health facility, COVID-19 risk perceptions and impact were significantly associated with health utilization (data not shown) and were therefore excluded from the final multivariate model. 
Table 3. Factors associated with households utilizing health care in the previous 4 weeks in Manicaland Province, Zimbabwe ( $=542$ ).

\begin{tabular}{|c|c|c|c|c|}
\hline & \multicolumn{2}{|l|}{ Univariate } & \multicolumn{2}{|l|}{ Multivariate } \\
\hline & OR [95\% Cl] & P-value & aOR $[95 \% \mathrm{Cl}]$ & P-value \\
\hline \multicolumn{5}{|l|}{ Age of respondent } \\
\hline $18-29$ years & Reference & & Reference & \\
\hline 30-39 years & $1.31[0.81,2.12]$ & 0.271 & $1.39[0.83,2.32]$ & 0.206 \\
\hline $40-54$ years & $1.00[0.63,1.59]$ & 0.998 & $1.43[0.86,2.38]$ & 0.165 \\
\hline$\geq 55$ years & $0.70[0.43,1.14]$ & 0.148 & $1.89[0.99,3.61]$ & 0.054 \\
\hline \multicolumn{5}{|l|}{ Marital status of respondent } \\
\hline Not married & Reference & & & \\
\hline Married/co-habiting & $1.29[0.89,1.89]$ & 0.182 & - & - \\
\hline \multicolumn{5}{|l|}{ Highest level of education attended by respondent } \\
\hline Primary school or lower & Reference & & Reference & \\
\hline Secondary school or higher & $1.88[1.32,2.68]$ & 0.001 & $2.16[1.36,3.43]$ & 0.001 \\
\hline \multicolumn{5}{|l|}{ Employment status of respondent } \\
\hline Employed & Reference & & Reference & \\
\hline Unemployed & $1.53[1.07,2.19]$ & 0.02 & $1.63[1.11,2.41]$ & 0.013 \\
\hline \multicolumn{5}{|l|}{ District } \\
\hline Buhera & Reference & & Reference & \\
\hline Makoni & $2.06[1.30,3.27]$ & 0.002 & $2.35[1.44,3.84]$ & 0.001 \\
\hline Mutare rural & $2.33[1.58,3.43]$ & $<0.001$ & $2.57[1.71,3.92]$ & $<0.001$ \\
\hline At least one child less than 5 years living in the household & $2.17[1.52,3.10]$ & $<0.001$ & $2.35[1.59,3.46]$ & 0.001 \\
\hline At least one pregnant woman living in the household & $2.42[1.42,4.15]$ & 0.001 & $2.22[1.26,3.93]$ & 0.006 \\
\hline \multicolumn{5}{|l|}{ Household size } \\
\hline$\leq 4$ household members & Reference & & & \\
\hline$>4$ household members & $1.32[0.93,1.85]$ & 0.117 & - & - \\
\hline \multicolumn{5}{|l|}{ Household wealth } \\
\hline Poorest & Reference & & & 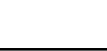 \\
\hline Middle & $0.90[0.59,1.35]$ & 0.599 & - & - \\
\hline Wealthiest & $1.42[0.94,2.15]$ & 0.100 & - & - \\
\hline
\end{tabular}




\begin{tabular}{|c|c|c|c|c|}
\hline & \multicolumn{2}{|l|}{ Univariate } & \multicolumn{2}{|l|}{ Multivariate } \\
\hline & OR $[95 \% \mathrm{Cl}]$ & P-value & aOR $[95 \% \mathrm{Cl}]$ & P-value \\
\hline Had health insurance & $1.15[0.49,2.70]$ & 0.749 & - & - \\
\hline Travel minutes to health facility & $1.00[0.99,1.01]$ & 0.372 & - & - \\
\hline \multicolumn{5}{|l|}{ Perceived problems at health facility } \\
\hline Availability of medicines at facility & $1.18[0.83,1.67]$ & 0.362 & - & - \\
\hline Cost of services or treatment & $1.28[0.83,1.98]$ & 0.259 & - & - \\
\hline Time waited to see a provider & $0.95[0.61,1.47]$ & 0.805 & - & - \\
\hline Availability of personnel at facility & $1.66[1.04,2.64]$ & 0.032 & - & - \\
\hline Treatment by staff & $1.89[1.18,3.04]$ & 0.008 & - & - \\
\hline Amount of explanation received about the problem or treatment & $1.16[0.70,1.92]$ & 0.577 & - & - \\
\hline Visual privacy of examination & $1.58[0.93,2.69]$ & 0.093 & - & - \\
\hline Auditory privacy of consultation discussion & $2.01[1.15,3.51]$ & 0.014 & - & - \\
\hline Hours of service at facility & $1.60[0.93,2.75]$ & 0.087 & - & - \\
\hline Ability to discuss problems or concerns about health & $1.22[0.72,2.09]$ & 0.461 & - & - \\
\hline Number of days services are available & $1.77[0.87,3.60]$ & 0.117 & - & - \\
\hline Cleanliness of the facility & $0.92[0.37,2.30]$ & 0.858 & - & - \\
\hline \multicolumn{5}{|l|}{ COVID-19 risk perceptions and impact } \\
\hline Concerned about COVID-19 spread in community & $1.44[1.03,2.02]$ & 0.034 & - & - \\
\hline Concerned about COVID-19 infection & $1.41[0.99,1.99]$ & 0.051 & - & - \\
\hline Loss of household income experience due to COVID-19 pandemic & $1.34[0.80,2.26]$ & 0.272 & - & - \\
\hline
\end{tabular}

OR: odds ratio. aOR: adjusted odds ratio. $95 \%$ CI: 95\% confidence interval. Significant P-values are bolded. - indicates variables not retained in the final model. 


\section{DISCUSSION}

Understanding the factors influencing health care utilization during the COVID-19 pandemic is crucial to responding to the COVID-19 pandemic and avoiding additional indirect deaths due to the pandemic. The present analysis sought to describe potential barriers to health care utilization and identify factors associated with utilization of health care services in Manicaland Province, Zimbabwe during the COVID-19 pandemic. Respondents perceived that the unavailability of medicines, the cost of services and the time waiting to see a provider as major problems at health facilities. Perceptions of health facilities, such as quality of care and availability of services and medications can impact perceived benefits from utilizing health care. Perceived poor quality of care has been identified as a determinant of poor utilization of health services in other settings. ${ }^{20}$ However, in the present study, indicating that treatment by health facility staff, auditory privacy and availability of personnel at the facility was a problem was associated with a greater odds of utilizing health care in the previous 4 weeks in the univariate analysis. However, after controlling for confounding, none of these factors were significantly associated with health care utilization. Households reporting recently visiting a health facility may have experienced these problems due to reductions in available health care workers and resources amid increases in COVID-19 cases and deaths. The second wave of COVID-19 cases in Zimbabwe spanned December 2020 to February 2021 and highlighted the fragility of the health care system, with hospitals reaching capacity, and depleted resources (Figure 1). ${ }^{29}$ Workers and resources, such as ventilators and personal protective equipment (PPE) are needed to effectively mitigate a COVID-19 surge, while ensuring health care worker and patient safety.

Despite concerns about the quality of care, more than half of households still reported using health services in the previous 4 weeks amidst the COVID-19 pandemic. Furthermore, households with a child less than 5 years of age or a pregnant woman, located in Makoni or Mutare rural districts, and respondents who had a higher education, were unemployed and were 55 years of age or older had a higher likelihood of health care utilization during the pandemic. Pregnant women and children require more frequent contact with the health system for services such as vaccinations, antenatal care, family planning and labor and delivery. According to the most recent Zimbabwe Demographic and Health Survey, 93\% of pregnant women attend at least one antenatal care (ANC) visit with a skilled provider, $77 \%$ of pregnant women delivered in a health facility and $78 \%$ of children received all 8 childhood vaccinations. ${ }^{30}$ The higher likelihood of health care utilization among households with pregnant women and children points to continued demand for these services despite the COVID-19 pandemic. Continued focus on maintaining access to and provision of these essential health services is crucial throughout Manicaland Province, particularly in Buhera district. Households in Makoni and Mutare rural districts were more likely to use health care services compared to Buhera district. Makoni and Mutare rural districts have more densely organized health facilities at all levels of care and a larger economically active population compared to Buhera district. ${ }^{26}$ Additionally, more people in Buhera attend Apostolic churches, which prohibit the use of western and modern medicine. Several studies have found reduced utilization of maternal and child health services among members of Apostolic churches in Zimbabwe. ${ }^{31,32}$

In the present study, most (96\%) households had no health insurance, cited affordability of services and treatment as a major barrier and experienced a partial or complete loss of income as a result of the pandemic. Efforts are needed to remove financial barriers and alleviate disparities in health care utilization. Households unable to afford treatment and services can have greater access when treatment and services are either free or at a reduced cost. In 2010, reforms to the Zimbabwean health system were first enacted to alleviate barriers to health care utilization among poor and rural households. ${ }^{33}$ Health care reforms such as results-based financing (RBF) should be prioritized during the COVID-19 pandemic. The pandemic has impacted livelihoods, and many are unable to work and face additional financial hardships. Implementation of RBF may include increased financial incentives to health facilities that meet certain targets set by the program, which ultimately drive down costs for patients. ${ }^{34}$ Incentives can also work to improve health facility perceptions of the community, through incentivizing quality of care.

This study found a higher odds of health care utilization among households where the respondent had a higher education and was older. Other studies in sub-Saharan Africa have similarly observed that those with higher attained education level had higher odds of health care utilization. ${ }^{10-12}$ Those who attended primary school or have no formal education may not perceive the need to seek care and may have less knowledge of COVID-19 and other diseases. ${ }^{34} \mathrm{~A}$ higher level of education promotes cues to utilize health care. The increased odds of health care utilization with increasing age is likely a manifestation of increased perceived susceptibility, cues to action and conviction that the individual can successfully execute health care utilization to achieve their desired outcome. ${ }^{9}$ The distribution of information concerning COVID-19 and health services should target households headed by younger and less educated individuals.

There are several limitations to this study. Potential biases in this study include non-response bias and recall bias. Non-response bias was addressed through the sampling process, while recall bias was addressed through the use of well-designed surveys and experienced interviewers. The cross-sectional design of this study facilitated the assessment of health care utilization at one point in time but precludes the assessment of trends over time and the inference of causality. Patterns of health care utilization typically vary throughout the year, especially for endemic diseases such as malaria and diarrhea. Therefore, the impact of the pandemic on health care utilization may be heterogenous and dependent on season. The results of this study are only generalizable to primarily rural areas in Zimbabwe. 
Research concerning the pandemic's impact on health facilities and households in any setting is very limited, therefore the findings of this study add to the body of evidence on health care utilization in the wake of the COVID-19 pandemic.

\section{CONCLUSIONS}

Here, factors associated with health care utilization in Manicaland Province, Zimbabwe amidst the COVID-19 pandemic were assessed. Future research should aim to examine health care utilization during different timepoints throughout the pandemic and include more urbanized areas in sub-Saharan Africa. Periods of stricter lockdown where schools and non-essential businesses were closed may have an even greater impact on the health care utilization, and comparisons of different timepoints and locations can provide useful information to promote health care utilization. Zimbabwe and many other African countries anticipate another surge in COVID-19 cases and deaths driven by the emergence of SARS-COV-2 variants. In response, several governments have re-instituted travel restrictions, lockdowns and other measures to curb transmission. ${ }^{35}$ To promote health care utilization, continuous education programs on COVID-19 infection, transmission, prevention and vaccinations should target those with less education, lower socioeconomic status and the elderly.

\section{ACKNOWLEDGEMENTS}

The research team would like to thank all respondents.

FUNDING

No external funding was received.

\section{AUTHORSHIP CONTRIBUTIONS}

JS performed the data analysis and drafted the manuscript. MK and OT conceived the study design, coordinated the study, and critically revised the manuscript. MSN and JM critically revised the manuscript. All authors reviewed and approved the final manuscript for publication.

\section{COMPETING INTERESTS}

The authors completed the Unified Competing Interest form at http://www.icmje.org/disclosure-of-interest/ (available upon request from the corresponding author), and declare no conflicts of interest.)

\section{CORRESPONDENCE TO:}

Mufaro Kanyangarara, Department of Epidemiology and Biostatistics, Arnold School of Public Health, University of South Carolina, 915 Greene Street, Columbia, South Carolina, 29201.nufaro@mailbox.sc.edu

Submitted: October 26, 2021 GMT, Accepted: December 28, 2021 GMT 


\section{REFERENCES}

1. Eurosurveillance Editorial Team. Note from the editors: World Health Organization declares novel coronavirus (2019-nCoV) sixth public health emergency of international concern. Euro Surveill. 2020;25(5):200131e. doi:10.2807/1560-7917.es.2020.2 $\underline{5.5 .200131 e}$

2. World Health Organization. Second Round of the National Pulse Survey on Continuity of Essential Health Services during the COVID-19 Pandemic: Interim Report, 22 April 2021. World Health Organization; 2021.

3. Jewell BL, Mudimu E, Stover J, et al. Potential effects of disruption to HIV programmes in subSaharan Africa caused by COVID-19: results from multiple mathematical models. Lancet HIV. 2020;7(9):e629-e640. doi:10.1016/s2352-3018(20)302 11-3

4. Weiss DJ, Bertozzi-Villa A, Rumisha SF, et al. Indirect effects of the COVID-19 pandemic on malaria intervention coverage, morbidity, and mortality in Africa: a geospatial modelling analysis. Lancet Infect Dis. 2021;21(1):59-69. doi:10.1016/s147 3-3099(20)30700-3

5. Roberton T, Carter ED, Chou VB, et al. Early estimates of the indirect effects of the COVID-19 pandemic on maternal and child mortality in lowincome and middle-income countries: a modelling study. Lancet Glob Health. 2020;8(7):e901-e908. doi:1 0.1016/s2214-109x(20)30229-1

6. Lone SA, Ahmad A. COVID-19 pandemic - an African perspective. Emerg Microbes Infect. 2020;9(1):1300-1308. doi:10.1080/22221751.2020.177 $\underline{5132}$

7. Gilbert M, Pullano G, Pinotti F, et al. Preparedness and vulnerability of African countries against importations of COVID-19: a modelling study. Lancet. 2020;395(10227):871-877. doi:10.1016/s0140-6736(2 $\underline{0) 30411-6}$

8. World Health Organization. World Malaria Report 2020: 20 Years of Global Progress and Challenges. World Health Organization; 2020.

9. Champion VL, Skinner CS. The health belief model. In: Glanz K, Rimer BK, Viswanath K, eds. Health Behavior and Health Education: Theories, Research, and Practice. Jossey Bass; 2008.

10. Muriithi MK. The determinants of health-seeking behavior in a Nairobi slum, Kenya. European Scientific Journal. 2013;9(8):1857-7881.
11. Begashaw B, Tessema F, Gesesew HA. Health Care Seeking Behavior in Southwest Ethiopia. PLoS ONE. 2016;11(9):e0161014. doi:10.1371/journal.pone.0161 $\underline{014}$

12. Zyaambo C, Siziya S, Fylkesnes K. Health status and socio-economic factors associated with health facility utilization in rural and urban areas in Zambia. BMC Health Serv Res. 2012;12(1):389. doi:10.1186/147 2-6963-12-389

13. Baltussen RM, Yé Y, Haddad S, Sauerborn RS. Perceived quality of care of primary health care services in Burkina Faso. Health Policy Plan. 2002;17(1):42-48. doi:10.1093/heapol/17.1.42

14. Kanyangarara M, Munos MK, Walker N. Quality of antenatal care service provision in health facilities across sub-Saharan Africa: Evidence from nationally representative health facility assessments. $J$ Glob Health. 2017;7(2):021101. doi:10.7189/jogh.07.021101

15. Delamou A, Sidibé S, Camara A, Traoré MS, Touré A, Van Damme W. Tackling the COVID-19 pandemic in West Africa: Have we learned from Ebola in Guinea? Prev Med Rep. 2020;20:101206. doi:10.1016/ j.pmedr.2020.101206

16. McQuilkin PA, Udhayashankar K, Niescierenko M, Maranda L. Health-Care Access during the Ebola Virus Epidemic in Liberia. Am J Trop Med Hyg. 2017;97(3):931-936. doi:10.4269/ajtmh.16-0702

17. Wilhelm JA, Helleringer S. Utilization of nonEbola health care services during Ebola outbreaks: a systematic review and meta-analysis. J Glob Health. 2019;9(1):010406. doi:10.7189/jogh.09.010406

18. Elston JWT, Moosa AJ, Moses F, et al. Impact of the Ebola outbreak on health systems and population health in Sierra Leone. J Public Health (Oxf). 2016;38(4):673-678. doi:10.1093/pubmed/fdv158

19. Parpia AS, Ndeffo-Mbah ML, Wenzel NS, Galvani AP. Effects of Response to 2014-2015 Ebola Outbreak on Deaths from Malaria, HIV/AIDS, and Tuberculosis, West Africa. Emerg Infect Dis. 2016;22(3):433-441. do i:10.3201/eid2203.150977

20. Sochas L, Channon AA, Nam S. Counting indirect crisis-related deaths in the context of a low-resilience health system: the case of maternal and neonatal health during the Ebola epidemic in Sierra Leone. Health Policy Plan. 2017;32(suppl_3):iii32-iii39. doi:1 0.1093/heapol/czx108 
21. Helleringer S, Noymer A. Magnitude of Ebola relative to other causes of death in Liberia, Sierra Leone, and Guinea. Lancet Glob Health. 2015;3(5):e255-e256. doi:10.1016/s2214-109x(15)701 $\underline{03-8}$

22. Dzobo M, Chitungo I, Dzinamarira T. COVID-19: a perspective for lifting lockdown in Zimbabwe. Pan Afr Med J. 2020;35(Suppl 2):13. doi:10.11604/pamj.2020.3 $\underline{5.2 .23059}$

23. Makoni M. COVID-19 worsens Zimbabwe's health crisis. Lancet. 2020;396(10249):457. doi:10.1016/s014 0-6736(20)31751-7

24. Mackworth-Young CRS, Chingono R, Mavodza C, et al. Community perspectives on the COVID-19 response, Zimbabwe. Bull World Health Organ. 2021;99(2):85-91. doi:10.2471/blt.20.260224

25. Bagcchi S. Stigma during the COVID-19 pandemic. Lancet Infect Dis. 2020;20(7):782. doi:10.10 16/s1473-3099(20)30498-9

26. Zimbabwe National Statistics Agency. Zimbabwe Population Census 2012. ZIMSTAT; 2013. Accessed October 22, 2021. http://www.zimstat.co.zw/wp-cont ent/uploads/publications/Population/population/cens us-2012-national-report.pdf

27. Zimbabwe Ministry of Health and Child Care. The National Health Profile 2014 Report. Ministry of Health and Child Care Zimbabwe; 2014. Accessed October 22, 2021. https://www.zimstat.co.zw/wp-con tent/uploads/publications/Social/Health/NHP-2014.p $\underline{\mathrm{df}}$

28. Zimbabwe Ministry of Health and Child Care. COVID-19 Daily Updates. Ministry of Health and Child Care Zimbabwe; 2020. Accessed October 22, 2021. http://www.mohcc.gov.zw/index.php?option=co $\mathrm{m}$ phocadownload \&view $=$ category \&id $=12$ \&Itemid $=7$ $\underline{40}$
29. Murewanhema G, Makurumidze R. Essential health services delivery in Zimbabwe during the COVID-19 pandemic: perspectives and recommendations. Pan Afr Med J. 2020;35(Suppl 2):143. doi:10.11604/pamj.supp.2020.35.143.25367

30. Zimbabwe National Statistics Agency, ICF International. Zimbabwe Demographic and Health Survey 2015: Final Report. Zimbabwe National Statistics Agency (ZIMSTAT) and ICF International; 2016. Accessed October 22, 2021. http://dhsprogra m.com/pubs/pdf/FR322/FR322.pdf

31. Ha W, Salama P, Gwavuya S, Kanjala C. Is religion the forgotten variable in maternal and child health? Evidence from Zimbabwe. Soc Sci Med. 2014;118:80-88. doi:10.1016/j.socscimed.2014.07.066

32. Gerede R, Machekanyanga Z, Ndiaye S, et al. How to Increase Vaccination Acceptance Among Apostolic Communities: Quantitative Results from an Assessment in Three Provinces in Zimbabwe. J Relig Health. 2017;56(5):1692-1700. doi:10.1007/s10943-01 7-0435-8

33. Zeng W, Lannes L, Mutasa R. Utilization of Health Care and Burden of Out-of-Pocket Health Expenditure in Zimbabwe: Results from a National Household Survey. Health Syst Reform. 2018;4(4):300-312. doi:10.1080/23288604.2018.15132 $\underline{64}$

34. Friedman J, Das A, Mutasa R. Rewarding Provider Performance to Improve Quality and Coverage of Maternal and Child Health Outcomes: Zimbabwe Results-Based Financing Pilot Program. The World Bank; 2016.

35. Madziva R, Murewanhema G, Dzinamarira T, Herrera H, Musuka G. Enhancing SARS-CoV-2 surveillance at ports of entry between South Africa and Zimbabwe due to anticipated increased human mobility during the festive period. Public Health in Practice. 2021;2:100215. doi:10.1016/j.puhip.2021.10 $\underline{0215}$ 